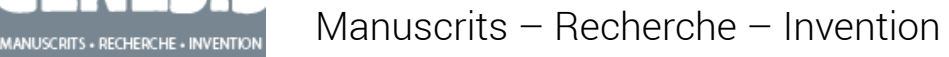

$51 \mid 2020$

Intertextualité - Exogenèse

\title{
La ressuscitée : Violette Leduc face à la censure, par Anaïs Frantz
}

\section{Véronique Montémont}

\section{(2) OpenEdition}

1 Journals

\section{Édition électronique}

URL : https://journals.openedition.org/genesis/5577

DOI : 10.4000/genesis.5577

ISSN : 2268-1590

\section{Éditeur :}

Presses universitaires de Paris Sorbonne (PUPS), Société internationale de génétique artistique littéraire et scientifique (SIGALES)

\section{Édition imprimée}

Date de publication : 15 décembre 2020

Pagination : $240-242$

ISBN : 979-10-231-0704-3

ISSN : 1167-5101

\section{Référence électronique}

Véronique Montémont, "La ressuscitée : Violette Leduc face à la censure, par Anaïs Frantz », Genesis [En ligne], 51 | 2020, mis en ligne le 10 janvier 2021, consulté le 11 janvier 2023. URL : http:// journals.openedition.org/genesis/5577 ; DOI : https://doi.org/10.4000/genesis.5577 
et la mise en lien de tout ce qu'un auteur a supprimé pendant son écriture ? De ce qu'il a ajouté, des remplacements effectués ? C'est à ce type de recensement, aboutissant à un dictionnaire des variantes, que s'est livré Jean-Louis Lebrave dès 1987 dans sa magistrale thèse d'État 11 . Il a analysé la dynamique des variantes en retraçant le parcours scriptural vers ce qui demeurera dans l'œuvre. C'est au trajet inverse que nous convie la perspective différentielle à l'œuvre dans l'ouvrage d'Authier-Revuz. Modifier son écrit, c'est à la fois circonscrire ce qui sera affiché comme de soi et rejeter le reste comme autre. Ce rejet, résultat d'une activité métadiscursive où la réflexivité, quoique non verbalisée, est mise en jeu de manière comparable à ce que révèle la modalisation autonymique, a pour objet un segment de discours qui, examiné pour lui-même, constitue une trace de cette altérité. L'étude de l'ensemble des mots rejetés pourrait ainsi permettre à la fois de retracer le geste de contour par lequel un scripteur délimite son discours, et de décrire l'ailleurs qu'il choisit d'éliminer et sur le fond duquel l'œuvre s'est élaborée.

La ressuscitée : Violette Leduc face à la censure

Anaïs Frantz (dir.), Violette Leduc. Genèse d'une ouvre censurée, Presses de la Sorbonne Nouvelle, Collection «Archives», 2016, 252 p.

Compte rendu

par Véronique Montémont*

Avec Violette Leduc, genèse d'une œuvre censurée, Anaïs Frantz et l'équipe des coautrices et coauteurs qu'elle a rassemblée autour d'elle apportent de nouveaux éclairages, infiniment précieux, sur une œuvre dont l'élaboration fut complexe, empêchée et souvent douloureuse. En effet, Leduc a durement vécu la censure qui a frappé Ravages (1955), une onde de choc qui résonnera dans toute son œuvre postérieure; si on y ajoute l'autocensure qu'elle s'appliquait, étroitement liée aux pressions sociales, et partant éditoriales, on comprendra pourquoi il est essentiel de (re)découvrir aujourd'hui cette œuvre à la lumière des études génétiques. S'inscrivant résolument dans les pas de Catherine Viollet, chercheuse pionnière des études leduciennes, et de Carlo Jansiti, biographe de Violette Leduc, le présent volume rassemble le fruit de plusieurs années de recherches du groupe Violette Leduc (ITEM-CNRS). Il s'agit bel et bien de « reconstruire Violette Leduc» (p. 15), depuis ses premiers pas en écriture, en montrant comment, au-delà de la question déterminante de son amitié avec Beauvoir, l'écrivaine s'inscrit en précurseure, au carrefour des problématiques genrées, sociales et sociétales qui émergent en France au lendemain de la Seconde Guerre mondiale. Son œuvre est une histoire d'émancipation, mais aussi de lutte contre la normalisation, qu'elle soit affective, sociale, sexuelle ou littéraire.

La première section de l'ouvrage, «Violette Leduc journaliste, les premiers écrits », nous permet de découvrir les textes publiés, dès 1940, dans le magazine Pour elle. Malgré l'extrême contrainte qui régit ces chroniques de mode et ces nouvelles, supposées exalter une forme rassurante et bourgeoise de la féminité dans la France de Vichy, on voit grâce à Alexandre Antolin comment Leduc parvient à sublimer l'exercice, nourrissant son écriture d'une poétique de la description intense, foisonnante et végétale. Ce vif intérêt pour la mode n'est au reste pas, comme le montre Kiev Renaud, une sujétion aveugle à ses diktats : car soigner son apparence, pour celle que Beauvoir appelait «la femme laide», équivaut à une prise de pouvoir, une façon de cacher des hontes secrètes ou son origine sociale. À l'époque où Leduc entre en écriture, le genre (au sens de gender) a une incidence majeure sur le positionnement des écrivaines femmes : comme le souligne Anaïs Frantz, Violette Leduc se trouve à la croisée des oppressions, «femme, mais aussi [...] bâtarde, fille de domestique, femme ayant avorté clandestinement, lesbienne» (p. 60), ce qui explique qu'elle subisse à ces titres multiples de plein fouet la censure qui frappe Ravages. L'Affamée, qui met en scène sa rencontre et son amitié avec Beauvoir, laisse voir par contrecoup à quel point la philosophe qui écrit en public, ne craignant ni les regards ni les critiques, offre à Leduc, qui redoute pour sa part l'exposition, le modèle précieux d'une femme capable de «transcender la condition féminine par le travail littéraire» (p. 64).

La deuxième partie du livre, «Violette Leduc à l'œuvre : la genèse de l'autobiographie», offre un exemple particulièrement réussi d'application de la lecture génétique à un ensemble d'écrits autobiographiques, dont le régime générique est déterminant pour la réception et la compréhension de l'œuvre. Leduc, travailleuse acharnée, n'a eu de cesse de récrire, parfois de sa propre initiative, parfois sous la direction de Beauvoir, parfois sous la contrainte de la censure, en usant de remaniements, de déplacements et de transpositions qui interrogent sur la nature générique de ses textes. Doit-on considérer ce remodelage du vécu, dont les manuscrits et notes préparatoires portent les traces essentielles, comme une trahison du pacte autobiographique? L'étude de Catherine Viollet, «L'écriture autobiographique à la lumière des manuscrits », démontre comment Leduc a travaillé à la conjonction des deux dimensions, fictionnelle et factuelle, qui ne s'annulent ni ne s'excluent. Des carnets et annotations marginales des manuscrits de La Bâtarde ont conservé la trace de vérifications, de tables de nombres, comme pour sans cesse «attester, en marge du récit, l'exactitude des faits relatés » (p. 72). Pour Catherine Viollet, ce besoin presque obsessionnel correspond au moment où l'écrivaine, souffrant d'un délire de persécution, tient à fournir la preuve qu'elle ne triche pas; en même temps, deux «je» coexistent, le biographique et le littéraire. C'est précisément

11. Jean-Louis Lebrave, Le Jeu de l'énonciation en allemand d'après les variantes manuscrites des brouillons d'Henri Heine, thèse de doctorat d'État, 1987, Université de Paris-Sorbonne.

* Université de Lorraine. 
la migration de l'un à l'autre, résultat, avant tout, d'une folle exigence stylistique qui pousse sans cesse à refaire et reprendre («Plus fiévreux, plus dramatique, plus précis - autrement», p. 73), que révèle cette lecture des cahiers. Un cahier manuscrit de L'Affamée, rédigé entre 1945 et 1947 , fournit à Mireille Brioude l'occasion d'étudier ce qu'elle nomme "l'effacement des frontières narratives » (p. 81). Beauvoir, objet d'une passion brûlante et non réciproque, qui sait être au centre du récit, a d'abord encouragé Leduc à tenir un journal. L'examen du cahier fait apparaitre de profonds remaniements du texte publié, à commencer par la suppression des repères datés qui l'ancraient dans le modèle diaristique. De même, les récits de rêve ne sont plus signalés comme tels, la plupart des noms propres disparaissent, ainsi que certaines scènes crues et annexes. Comme le confirme la réécriture de l'explicit, il s'est agi de «dire l'amour pour un être» (p. 98), dans sa nature absolue; le prix à payer, et qui a pu parfois dessécher le lyrisme du texte original, est un travail d'élagage, de déréférentialisation, et d'épure, propre à ouvrir la voie à «l'écriture de la violence amoureuse» (p. 99). L'archive va révéler comment l'écriture se charge de symboles : la «chemise orange» qui dans La Folie en tête abrite le manuscrit confié à Beauvoir déplie sous la plume d'Anaïs Frantz ses significations, qui confondent écriture et peau. Enfin, l'étude de genèse permet de lever des contresens, tel celui qui plane sur les derniers feuillets de La Chasse à l'amour, que Beauvoir a relu, corrigé et édité à titre posthume. Il a suffi d'une erreur dans la composition d'une date pour que l'explicit, disposé comme un poème en prose, acquière une valeur symbolique et presque testamentaire, là où il avait été conçu par son autrice comme un texte de transition.

La troisième partie du livre, «Reconstruire Ravages : censure éditoriale et génétique textuelle», va s'attacher à mettre en lumière les conséquences de métamorphoses - choisies ou imposées - du texte, notamment en ce qui concerne Ravages, dont on sait à quel point l'amputation fut un traumatisme majeur pour Leduc. La scène inédite du taxi, fortement édulcorée par Gallimard, est analysée de manière passionnante par Alexandre Antolin sous l'angle des échanges économico-sexuels. La narratrice, à qui Marc a offert restaurant et orchestre tzigane, devra le payer par une fellation imposée dans un taxi, c'est-à-dire un viol. Mais les deux protagonistes y ont des rôles instables et peu hétéronormés, tour à tour «féminin ou masculin, dominant et dominé» (p. 154) et la pratique sexuelle imposée parait subversive pour l'époque, ce qui a pu valoir l'affadissement de sa description. Ces scènes de viol, comme le montre Kaliane Ung, ponctuent l'œuvre leducienne, avec un tropisme récurrent, celui de la « compassion [de Violette Leduc] pour ceux qui la maltraitent» (p. 166). La blessure de la chair forcée se prolonge, chez l'écrivaine, au moyen d'un «glissement de terrain de la chair au texte» (p. 170), qui fait de la censure le prolongement de cette violence physique. C'est l'écriture, la réécriture plus exactement, qui rédime le double outrage : retravailler inlassablement le récit des abus ou les textes étouffés par la censure renverse la position victimaire, devient une façon de domestiquer et dominer l'événement - on pourrait presque ici parler d'empowerment de l'écriture.

L'étude des manuscrits fait mieux percevoir combien celle-ci, pour Violette Leduc se confond, magnifiquement, avec la chair, engrammant l'expérience érotique au plus profond de ses strates. Le personnage reparaissant d'Isabelle, qui emblématise l'amour, «l'amour de l'amour et même l'amour de l'écriture» (p. 181) infiltre toutes les couches de l'œuvre et l'irrigue : la scène de mariage hétérosexuel de Ravages étudiée par Alison Péron se révèle ainsi être une réécriture de la scène des noces fantasmée entre les deux femmes, retirée de La Bâtarde, mais publiée dans Thérèse et Isabelle.

La quatrième et dernière section, «Portraits cachés. Sur quelques feuillets retranchés », questionne enfin la représentation de soi et d'autrui, et surtout son retravail par le texte. La formule «une sentinelle aux portes de la littérature », conservée dans La Folie en tête, est l'écume d'autres images, initialement plus dévalorisantes : celle d'une femme «naïve», «fixe», qui regarde Sartre et Beauvoir écrire sans oser franchir le seuil du café. Relisant ces reformulations successives au prisme des travaux de Didier Eribon, Anaïs Frantz met en lumière un puissant complexe de classe, celui d'une transfuge, qui se sent illégitime, tant au regard de la filiation que de celui de la littérature. Le portrait de Sartre, avec son strabisme, a été resserré et atténué; non tant pour épargner le philosophe mais parce que l'écriture leducienne «s'envisage dans une réécriture permanente», comme le dit plus loin Olivier Wagner (p. 211), qui va en juguler le flot et le foisonnement initial. La réécriture des portraits de Nathalie Sarraute, qui apparaît à trois reprises dans La Folie en tête, masque, derrière la fascination intellectuelle avouée dans la version finale pour cette «droguée de littérature» (p. 216), la dimension érotique, pour Leduc, de cette amitié, occultée dans la version finale - l'écrivaine ayant dû faire un choix entre Sarraute et Beauvoir après leur brouille. Autre constat essentiel : les erreurs de dates commises par l'auteur qui révèlent combien, dans le domaine autobiographique, le souvenir ne se déploie pas en ligne droite. L'effort d'authenticité, permanent chez Leduc, coexiste avec une forte dynamique de «reformulations, de contournements ou même d'effacements » (p. 219), selon la formule d'O. Wagner qui invite à repenser nos attentes en termes de «vérité» quant à ce type d'écrits. Portrait de femmes, enfin, qui disparaissent au gré des nombreux feuillets supprimés de La Chasse à l'amour, comme Mireille Brioude en fait le constat : pourtant, quand on l'envisage dans sa configuration initiale, cette «constellation» (p. 232) féminine, où défilent Berthe, la mère, Fidéline, la grandmère, une voisine, Odette, replace Leduc au cœur d'une interrogation essentielle sur sa relation avec les femmes de sa vie, son non-désir de maternité et l'avortement qui interroge, là encore, la «portée symbolique de l'autobiographie» (p. 233). 
Signalons enfin la part belle, et tellement justifiée, que le volume fait à Catherine Viollet, dont on retrouvera avec émotion des pages de notes prises durant ses travaux sur Leduc, accompagnées d'un hommage rendu par ses amies Marie-Paul Andréo, Danielle Prévôt et Suzette Robichon, et de plusieurs documents présentés lors de l'exposition qui lui rendit hommage. Il semble également important de mentionner l'abondance et la qualité des nombreux et riches fac-similés de manuscrits, assortis de transcriptions, dont l'intérêt est rehaussé par une mise en page magnifique et une composition impeccable, celles d'un beau livre autant que d'un ouvrage savant.

Descendant strate après strate dans les profondeurs de l'écriture leducienne, dépliant sa multiplicité, déchiffrant avec subtilité et perspicacité les problématiques posées par cette œuvre exceptionnelle et son processus d'élaboration, ce volume collectif, qu'on lit comme un roman, est un véritable vademecum. Il sera indispensable à qui souhaite mieux connaître et comprendre une des figures majeures de la littérature française de la seconde moitié du $\mathrm{xx}^{\mathrm{e}}$ siècle; une femme unique qui sut inverser et renverser les forces de la bâtardise, l'hétéronormativité, la censure et la violence dans une œuvre au souffle et au lyrisme étincelants. 